\title{
Electronically Reconfigurable Phase Shifter for Reflectarray Applications at Microwave Bands
}

\author{
Pablo Padilla , Juan F. Valenzuela-Valdés , José L. Padilla , José M. Fernández-González , Manuel Sierra-Castañer
}

\begin{abstract}
This contribution provides the design of a microwave phase shifter for active steerable reflectarray devices at microwave frequencies. The device is based on a $3 \mathrm{~dB}$ hybrid coupler with three of its ports connected to reflective circuits. This reflective circuit is responsible of the phase variation by means of the use of an electrically controllable microwave varactor. More than $360^{\circ}$ phase shifting is obtained with this device as the signal is conducted through the device towards the reflective circuits in three different stages. The input port of the device is also the output one, providing the desired phase shifting and the reflective effect. This device is of great interest in reflectarray applications in order to provide controllable phase shifting at each array element. The document includes the design of the different parts of the shifter along with the device design. Results, considerations and discussion are also provided in this work.
\end{abstract}

Index Terms - reflectarray antenna, reflective phase shifter, microwave band, active antennas.

\section{INTRODUCTION}

Satellite communication systems have experienced a considerable growth the last decades, mainly due to the amount of telecommunication services and necessities that have been developed [1]. All this development has been translated into more amore demanding operating requirements for the communication system devices. In the case of the radiating terminals in wireless communication systems, these demanding requirements are related not only to operating issues: radiation pattern, directivity, gain, side lobe level, polarization purity, copolar/crosspolar ratio, etc. but also to mechanical and physical issues: size, compactness, profile, thermal operating range, etc.

In the case of satellite communications, phased array antennas such as reflectarrays $[2,3]$ and transmitarrays $[4,5]$ are becoming a realistic option to replace reflector-type antennas providing a quite similar radiating behavior and performance than conventional reflector-based designs, and overcoming strict requirements in terms of size, weight and low-profile. In the particular case of the reflectarray structures, the effect of transforming the feeder spherical wave into a planar standing wave is achieved by means of a reflecting array surface that introduces the proper phase delay at each element of the array. Thus, the reflectarray re-defines the reflected standing wave phase configuration so that the original feeder pattern is converted into a new different pattern. Fig. 1 provides a visual insight of the reflectarray concept. As it is depicted, the steering direction and pattern aspect ratio (Side lobe levels, main beam width, etc.) can be controlled tuning the transmission phase and amplitude of the radiated signal of each array radiating element [2].

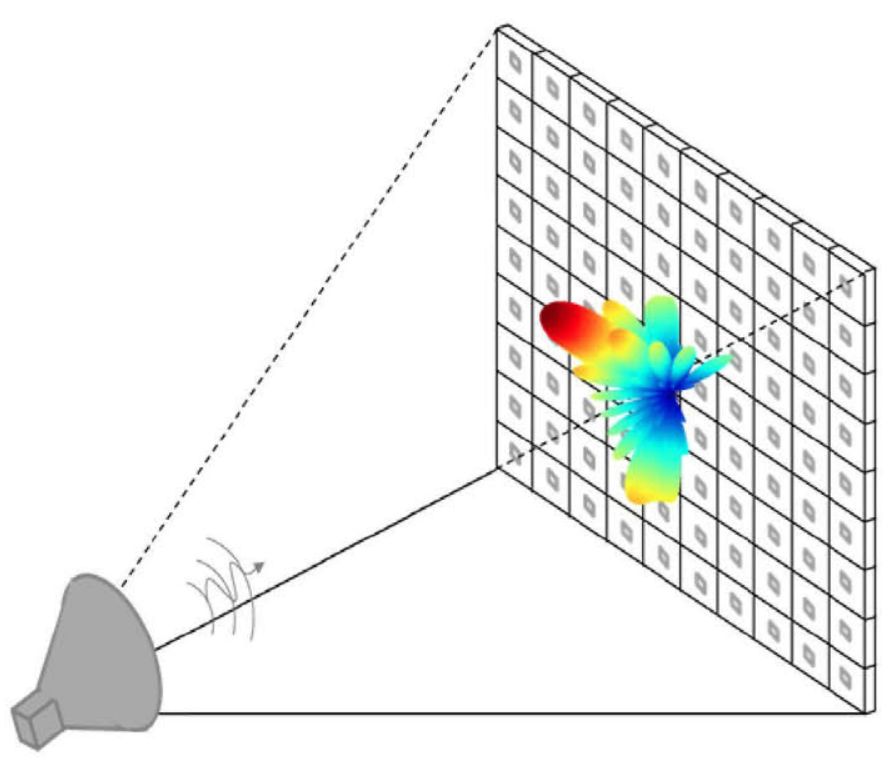

Fig. 1. Reflectarray scheme and working principle.

In the case of passive devices, the final features are fixed and cannot be modified after the design $[2,6]$. However, in the case of active devices, the radiation pattern and main lobe pointing direction can be controlled if the phase behaviour of each cell is somehow modifiable. This paper presents an electronically tunable phase shifter for reflectarray devices, based on reflective circuits. The tuning effect is introduced by the use of varactors.

This document is organized as follows: Section II provides the phase shifter working principle. Section III presents the phase shifter design and performance. Finally, conclusions are drawn in Section IV.

\section{Phase ShIFTER WORKING PRINCIPLE}

The phase shifter working principle is intimately related to the necessity of designing a reflective device due to the 
reflecting behavior of the reflectarray systems in which this shifter is intended to be added. In this way the input port of the shifter must also be the output one. The phase shifter is based on a modified 4-port $3 \mathrm{~dB}$ coupler. Considering port 1 the input port, ports 3 and 4 the $3 \mathrm{~dB}$ output ports with a difference of $90^{\circ}$ and port 2 the isolated port (see Fig. 2), the complete shifter functioning is as follows: the input signal in port 1 is derived towards ports 3 and 4 with a phase difference of $90^{\circ}$. In those ports the signals are delayed according to the reflective circuit configuration (first shifting stage) and sent towards port 2 , where the signals coming from ports 3 and 4 are combined in phase (the $90^{\circ}$ difference is reverted) and the resulting signal is again delayed due to the reflective circuit (second shifting stage). Then, the signal is redirected once more towards port 3 and 4, where again a phase variation is added (third shifting stage), and finally sent towards port 1 as the output of the device. This configuration is especially suitable for systems that require reflective devices, such as reflectarray structures.

\section{PHASE SHIFTER DESIGN AND PERFORMANCE}

This section provides the design of each constituting element together with the complete device design.

\section{A. Hybrid coupler}

The basic constituting element of the tunable phase shifter is a $3 \mathrm{~dB} / 90^{\circ}$ coupler, conveniently modified. The coupler is printed over the upper side of a Neltec substrate $\left(\varepsilon_{r}=2.17\right.$, $h=0.508 \mathrm{~mm}, \tan \delta=0.0002$ ), whereas the lower side is a copper ground plane. Figure 2 provides the hybrid coupler model. The simulation results are provided in Fig. 3.

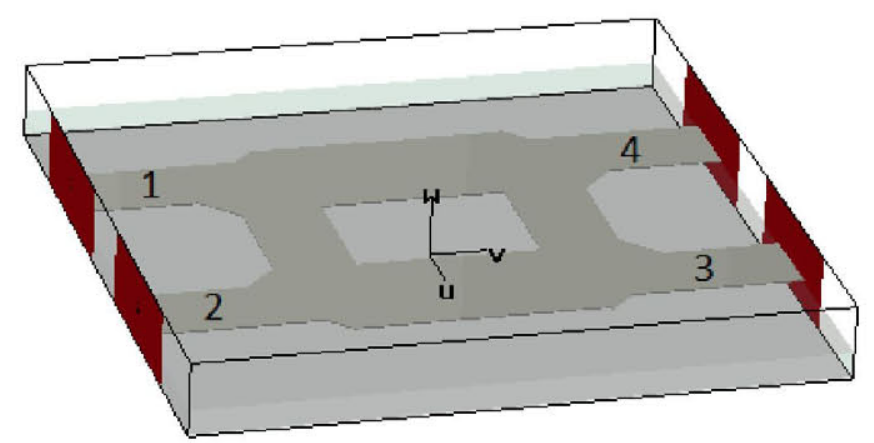

Fig. 2. Hybrid coupler design model.

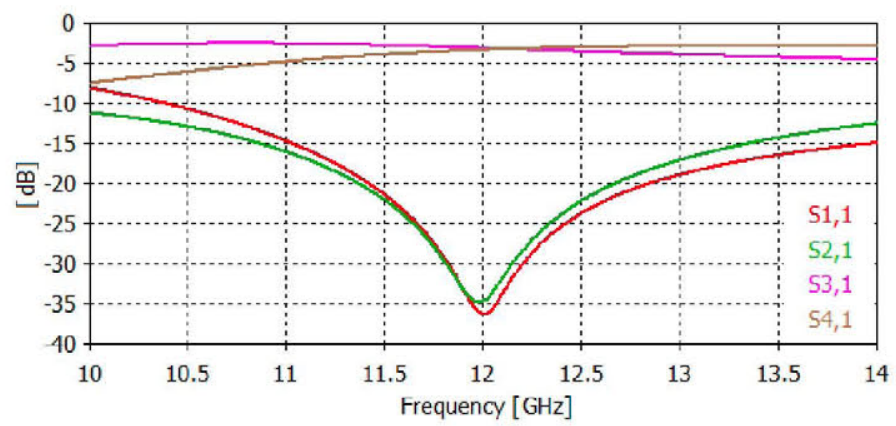

Fig. 3. Hybrid coupler design results.
As it is depicted, the working frequency is centered at 12 $\mathrm{GHz}$ and the bandwidth can be assumed to have more than $1.2 \mathrm{GHz}$ at $-20 \mathrm{~dB}$ in $\mathrm{S}_{11}$ level (more than $4 \mathrm{GHz}$ if a $-10 \mathrm{~dB}$ reference level of $S_{11}$ is considered). Additionally the symmetry of the complete device guarantees that the same signal level is achieved at ports 3 and 4 with a precise $90^{\circ}$ difference between signals in those ports. Additionally, the isolation purity in port 4 is of the same level than the matching level at port 1 .

\section{B. Reflective RF circuit}

The additional element of phase shifter is a reflective RF circuit formed by an equivalent LC circuit with a printed inductance and a varactor with variable capacity value. Figure 4 provides the reflective RF circuit model. As it is shown, the inductance is provided by a thin strip joined to the varactor, while the connection to the ground plane is obtained through a metallic via.

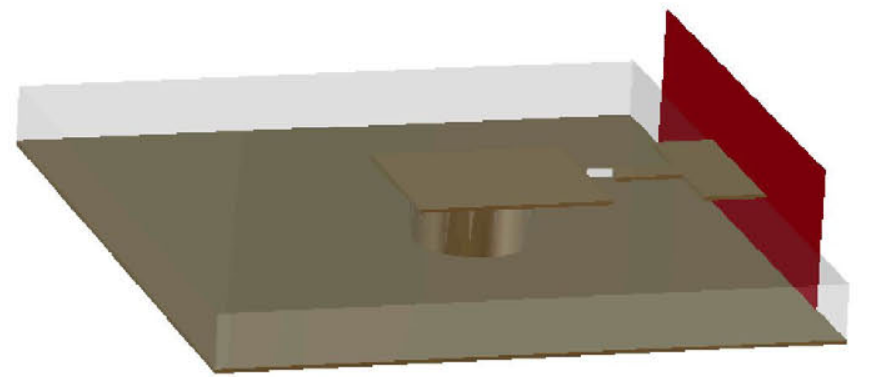

Fig. 4. Reflective RF circuit design model.

The simulation results of the reflective circuit are provided in Fig. 5. As it is shown a phase variation of $\sim 200^{\circ}$ is achieved. It is remarkable that the phase variation is not linear with the capacity value. However the same effect is observed when studying the specification sheet of the varactor regarding voltage and capacity value. Hopefully, when both behaviors are combined, the final phase shift versus voltage shows its linearity in almost all the capacity range of the varactor. In particular the linearity is maintained between $0.13 \mathrm{pF}$ and $1.95 \mathrm{pF}$.

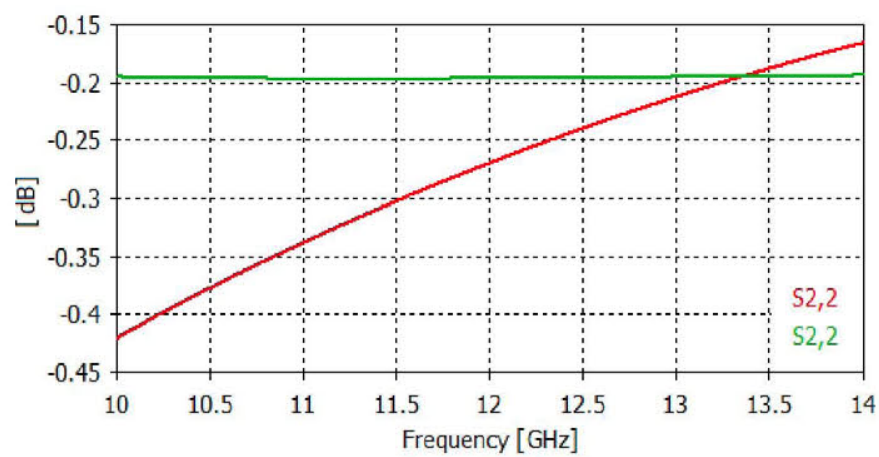

a) 


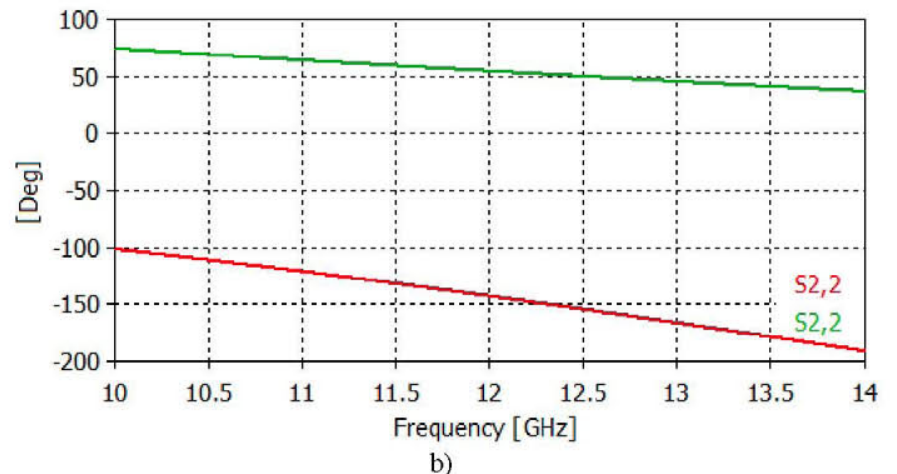

Fig. 5. Reflective RF circuit design results $(\mathrm{Cmin}=0.13 \mathrm{pF}$, red line; $\mathrm{C} \max =2.2 \mathrm{pF}$, green line), a) amplitude, b) phase

As it is seen, in the complete capacity range $(0.13$ to $2.2 \mathrm{pF})$ the total phase variation is of around $200^{\circ}$. Considering the linear range, the phase variation is reduced to around $172^{\circ}$.

\section{Complete phase shifter integration}

In the complete device, port 1 is preserved, whereas ports 2, 3 and 4 are connected to reflective circuits, yielding the triple phase variation of the signal in its way through the phase shifter. Figure 6 provides the complete phase shifter model.

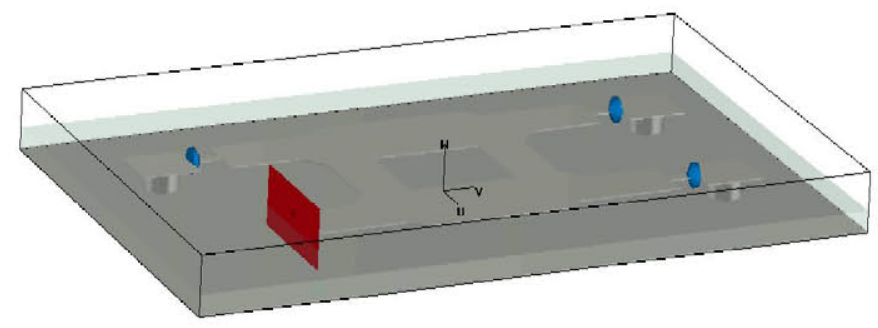

Fig. 6. Complete phase shifter design model.

The simulation results of the complete shifting device are provided in Fig. 7.

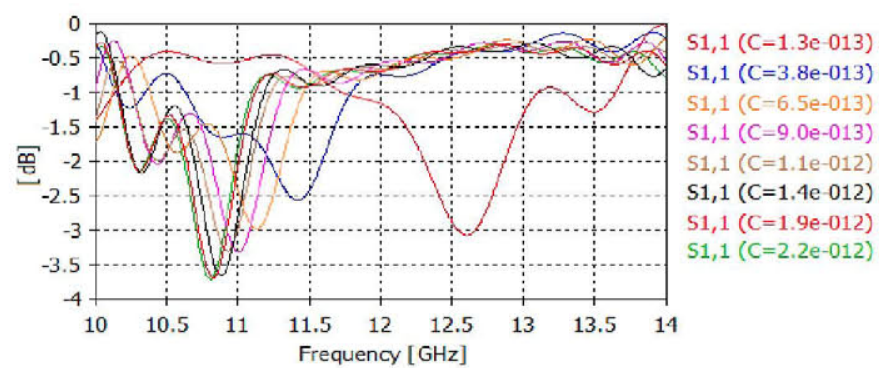

a)

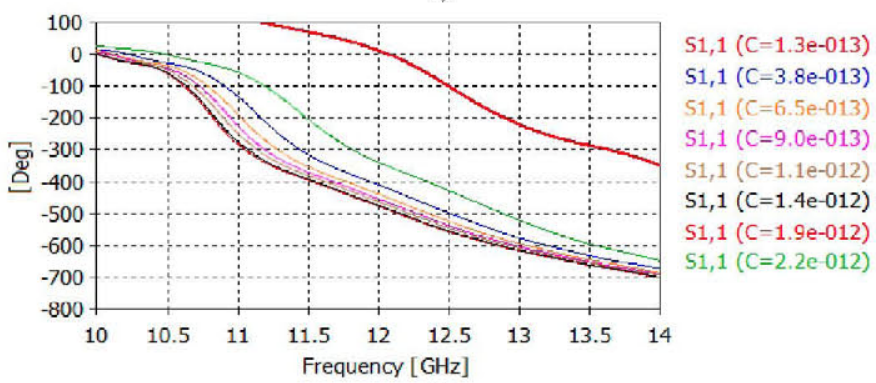

b)

Fig. 7. Complete phase shifter design results: a) amplitude, b) phase.
As it was mentioned, the input signal in port 1 is derived towards ports 3 and 4, re-sent towards port 2, again redirected once more towards port 3 and 4, and finally sent towards port 1 as the output of the device, obtaining three times the phase delay provided by each reflective circuit. The shifter presents low loss levels in the working band (between $0.5 \mathrm{~dB}$ and 1.5 $\mathrm{dB}$, the last one in the worst case). The shifter provides a phase shifting capability of around $470^{\circ}$, much higher than the $360^{\circ}$ needed to have complete phase variation (considering phase wrapping). Additionally, the shifter provides compactness and a reduction in the number of lumped elements, compared to other designs available in the literature. The size of the shifter fits in an array structure even if the distance between cells is lower than half a wavelength in the substrate $\left(\lambda_{\mathrm{g}}\right)$.

\section{CONCLUSION}

This paper provides the design of a phase shifter for active reflectarray systems at microwave frequencies. The device, based on a $3 \mathrm{~dB} / 90^{\circ}$ coupler, has three of its ports connected to electronically controllable reflective circuit which contains varactors. The complete device provides more than $360^{\circ}$ phase shift $\left(475^{\circ}\right.$ in the design) with only one port acting simultaneously as input and output port. Considering the worst case, the circuit losses are around $2 \mathrm{~dB}$ and the phase shifting maintains linearity in terms of phase shift versus polarization voltage. The complete phase shift is obtained sequentially in three different stages as the signal goes through the shifter. This device is of great interest in reflectarray applications as it may provide variable phase shifting at each array element.

\section{ACKNOWLEDGMENT}

The electromagnetic design has been developed with CST Studio under license agreement between CST and Technical University of Madrid. This work is supported by the TEC2014-55735-C3-1-R of the Spanish National Program of Research, Development \& Innovation and by the European Community's Seventh Framework Programme Marie Curie Action under Grant No. 291780 (Andalucía Talent Hub).

\section{REFERENCES}

[1] M.A. Salas Natera, A. Garcia Aguilar, J. Mora Cuevas, J.M. Fernandez, P. Padilla de la Torre, J. Garcia Gasco Trujillo, R. Martinez Rodriguez Osorio, M. Sierra Pérez, L. de Haro Ariet and M. Sierra Castañer, Satellite Communications, chap. 7 "New Antenna Array Architectures for Satellite Communications", pp. 167-194, InTech, July 2011.

[2] J. Huang, J.A. Encinar, Reflectarray Antennas, Wiley-IEEE Press, 2008.

[3] J.A. Encinar and J.A. Zornoza: "Three-layer printed reflectarrays for contoured beam space applications", IEEE Transactions on Antennas and Propagation, Vol. 52, No. 5, pp. 1138-1148, 2004.

[4] Padilla, A. Munoz-Acevedo, and M. Sierra-Castaner, "Passive planar transmitarray microstrip lens for microwave purpose," Microwave \& Optical Tech. Lett., vol. 52, no. 4, pp. 940-947, Feb 2010.

[5] P. Padilla, A. Muñoz-Acevedo, M. Sierra-Castañer and M. Sierra-Pérez, "Electronically reconfigurable transmitarray at $\mathrm{Ku}$ band for microwave applications", IEEE Transactions on Antennas and Propagation, Vol. 58, No. 8, pp. 2571-2579, 2010.

[6] J.L. Padilla, P. Padilla, J.F. Valenzuela-Valdés and J.M. FernándezGonzález, "High-frequency radiating element and modified $3 \mathrm{~dB} / 90^{\circ}$ electronic shifting circuit with circular polarization for broadband reflectarray device cells," Electronics Letters, Vol.50, No.15, pp.10421043,2014 